\title{
La falacia del poder de la demanda
}

Paul Alvarado*

Introducción

A mediados del año 2002 surgió una iniciativa conjunta del sector industrial, representado por la Asociación Salvadoreña de Industriales (ASI), el Ministerio de Economía y el proyecto de Energía Eléctrica del Istmo Centroamericano ( PREEICA ) denominada El poder de la demanda, que consiste en la promoción, por medio de asesores en ahorro energético capacitados por el Instituto Tecnológico Centroamericano (ITCA), de la reducción del consumo de energía eléctrica sobre todo en las horas de mayor demanda (de 11:00 a 14:59 y de 18:00 a 21:59) ${ }^{1}$ con el objeto de reducir el precio promedio del Mwh en el mercado mayorista de energía. La apuesta del sector industrial y del Ministerio de Economía es que al reducir la demanda de energía, siguiendo la lógica de oferta y demanda, el precio promedio del kilovatio horas (Kwh) se reduzca. Esto debido a que no entrarían en funcionamiento las unidades generadores a base de combustible que son las que tiene los precios (y costos variables) más altos y que generalmente determinan el precio del mercado. A primera vista parece una estrategia viable; sin embargo un poco de detenimiento y otro tanto de teoría económica pueden mostrar que el poder de la demanda no es tal bajo

* Magíster en Economía, Pontificia Universidad Católica de Chile. Ingeniero Electricista por la Universidad Centroamericana "José Simeón Cañas". 
ciertas circunstancias especiales. Dichas circunstancias tienen buenas probabilidades de existir en El Salvador y de eso tratará este artículo.

\section{El mercado eléctrico en El Salvador}

\subsection{Antecedentes}

La actual Ley General de Electricidad existe desde $1996^{2}$. En ese entonces, el sector eléctrico era manejado fundamentalmente por el Estado a través de la Comisión Ejecutiva Hidroeléctrica del Río Lempa (CEL). Ya se había permitido el ingreso de un generador privado ${ }^{3}$, pero sólo como proveedor de CEL bajo un contrato a largo plazo. Sin embargo, no fue sino hasta 1997 cuando se permitió a los primeros inversores privados participar libremente en el mercado con la separación y venta de las empresas distribuidoras (CAESS, EEO, DEUSEM, CLESA y CLESS). Luego en 1998, la CEL separa las actividades que desarrollaba hasta ese momento (Generación Hidráulica, Generación Geotérmica, Generación Térmica, Transmisión y la Coordinación del Sistema) y vende a privados la generación Térmica, crea la empresa Transmisora de energía y la coordinación del sistema se entrega a la Unidad de Transacciones (UT) en la que tienen participación todos los agentes del mercado mayorista.

A partir de ese momento el mercado eléctrico se convierte en un mercado libre y con, relativamente, poca intervención estatal.

\subsection{La operación del mercado}

La Ley General de Electricidad busca generar competencia en el mercado de generación de energía. En ese orden de cosas se organizaron dos mercados, uno, el Mercado de Contratos, donde los participantes de mercado celebran contratos de forma libre, y otro, el Mercado Regulador del Sistema (MRS), donde se compra y vende energía de oportunidad como si se tratara de una bolsa de energía donde la interacción directa entre la oferta y demanda determina el precio del mercado.

En el MRS, la Unidad de Transacciones (UT) despacha primero la energía de las plantas que ofertaron el precio más bajo, y continúa despachando las unidades en orden ascendente de precios hasta que se suple exactamente la potencia demandada. Idealmente las últimas plantas en ser despachadas debiesen ser las plantas térmicas o que funcio- 
nan a base de combustible; esto es así porque, debido a sus altos costos variables, ofertarían precios relativamente altos ${ }^{4}$. El precio de mercado del megavatio/hora (MWh) queda determinado, entonces, por el precio que ofertó la última máquina en entrar en línea (la unidad marginal, en términos formales). Luego, al ir cambiando la demanda también cambiará el precio de la energía. La UT calcula el precio de la energía en una base horaria de forma que existen 24 precios en un día ${ }^{5}$.

\subsection{Reducción de demanda vrs. respuesta de la demanda}

Existe una diferencia sustancial entre una reducción de la demanda de energía por una vez y la respuesta de la demanda ante las variaciones de precio. De la teoría microeconómica básica sabemos que una reducción de la demanda es un descenso del nivel demandado(Q) de energía a cualquier nivel de precio $(\mathrm{P})$. Es decir que la reducción obedece a factores distintos al precio y cuando eso ocurre la curva de demanda (D-D) se desplaza hacia la izquierda.(D'-D') (figura 1a)

Diferente es el caso de cambios en la cantidad demandada de energía debidos a cambios en el precio de esta. Al igual que ocurre con cualquier bien, la cantidad demandada de éste cae cuando sube el precio y aumenta cuando el precio cae (Figura 1b). Un indicador de la Respuesta de la Demanda ante variaciones de precio es la "Elasticidad Precio", que se define como la variación porcentual de la cantidad demandada ante una variación porcentual unitaria del precio.

Matemáticamente:

donde:

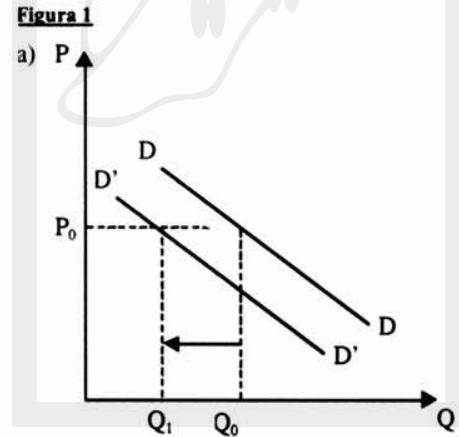

Q: cantidad demandada

P: precio.

b)

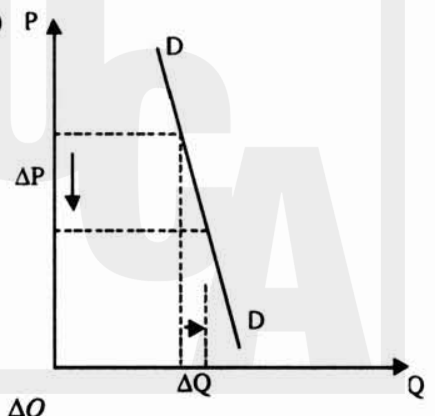

$\Delta Q$

$\eta=\underset{P P}{Q} \underset{P}{Q} \approx \operatorname{dLn} Q=0$ 
Cuanto más sensible sea la demanda al precio (curva de demanda más horizontal) más alto será el valor de la elasticidad y mayor será la variación en precio ante una variación en la cantidad demandada.

Cuando los industriales se proponen reducir su consumo energético global usando iluminación eficiente, aislamiento térmico, equipos de acondicionamiento de ambientes ahorradores de energía, etc. están reduciendo la demanda a cualquier nivel de precios (retroceden la curva de demanda de corto plazo). Cuando los industriales colocan dispositivos que desconectan carga a las horas de mayor precio de la energía, cuando acomodan sus procesos para que consuman más energía en el momento en que el precio es más bajo, entonces su demanda está respondiendo a la variación de precio (movimiento a lo largo de la curva) ${ }^{6}$.

Ambos movimientos tienden a reducir el precio de mercado de un bien tal como se muestra en la figura 2 .
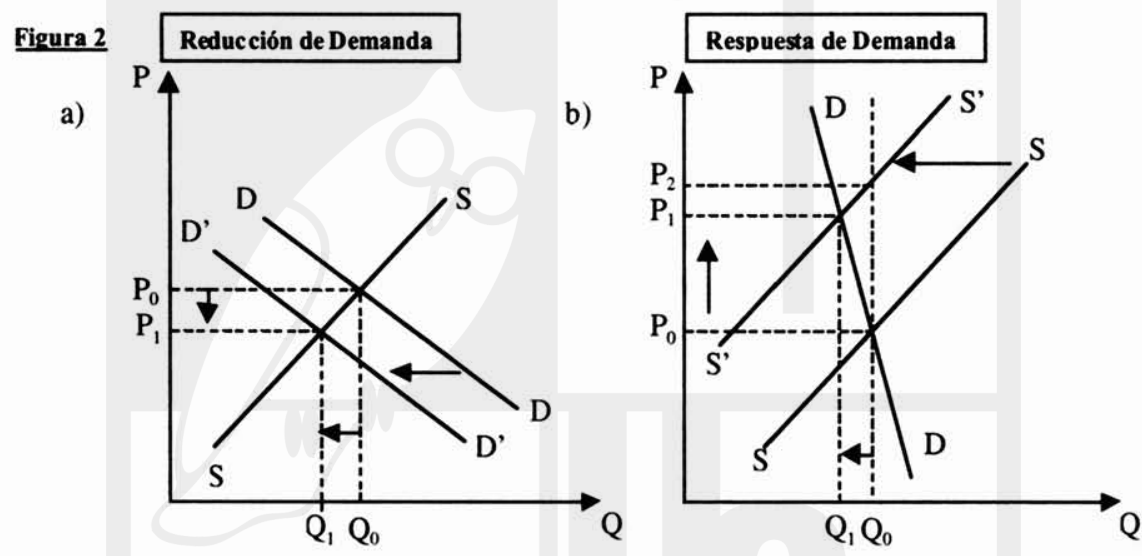

En la figura 2a puede verse que una reducción de la demanda (movimiento de la curva D-D hacia la curva D'-D'), dado un nivel de oferta (curva $S-S$ ), hace que el precio en el mercado caiga de $P_{0}$ a $P_{1}$ y que la cantidad demandada caiga de $\mathrm{Q}_{0}$ a $\mathrm{Q}_{1}$. En la figura $2 \mathrm{~b}$ puede verse que ante una reducción de la oferta (desplazamiento de la curva S-S hacia la curva $S^{\prime}-S^{\prime}$ ), la cantidad demandada se reduce de $Q_{0}$ a $Q_{1}$ y el precio de sube de $P_{0}$ hasta $P_{1}$. Sin embargo, en este último caso, el precio llegó tan solo hasta $P_{1}$ debido a la respuesta en demanda; si la 
demanda no hubiese reaccionado y la cantidad demandada hubiese seguido siendo igual a $Q_{0}$ el precio habría subido hasta $P_{2}$ después de la contracción de la oferta. Desde luego, cuanto mayor sea la elasticidad de la demanda (mayor sensibilidad de la demanda ante cambios de precio) menor será el precio, $\mathrm{P}_{1}$, que resultará de la disminución de la oferta.

Figura 3

a)

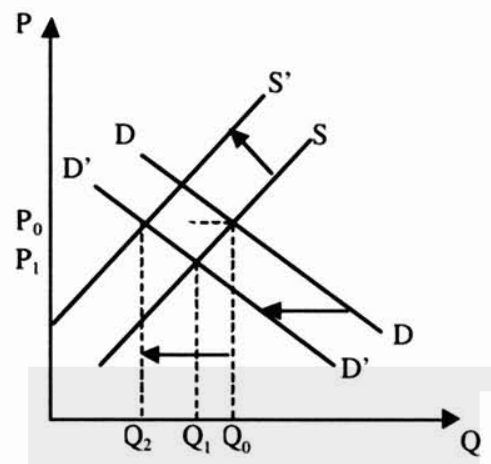

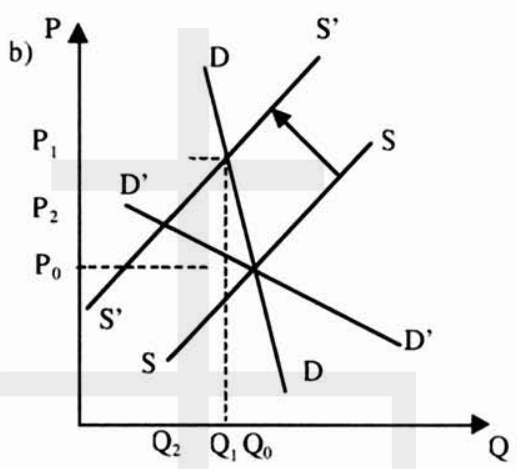

Ahora bien, que ocurra una reducción de la demanda de energía no es garantía de que bajen los precios. Esto es así debido a que existe la posibilidad de que se reduzca, simultáneamente, la oferta eléctrica. Otro factor importante es el grado de respuesta de la demanda ante cambios en el precio. Las demandas muy inelásticas experimentarán mayores sobreprecios que demandas más elásticas, para los mismos cambios en la oferta. Lo anterior se explica mejor en la figura 3.

Como se observa en el Figura 3a, una vez que la curva de demanda se contrae (de la curva D-D a la curva D'-D'), se reduce la cantidad demandada de $Q_{0}$ a $Q_{1}$ y el precio tiende a ser igual a $P_{1}$. Si ahora la oferta retrocede lo suficiente (de la curva S-S a la curva S'-S') puede lograr que la cantidad demandada sea $Q_{2}$ y el precio vuelva a ser $P_{0}$.

En el gráfico $3 b$, sin embargo, se aprecia que siempre que la demanda tenga algún grado de respuesta a las variaciones de precio (elasticidad precio $=\eta>0$ ) las contracciones de la oferta no se traducirán, en su totalidad, en aumentos de precios. Esto es así debido a que solo una parte del recorte de oferta se convierte en aumento de precio, la otra parte se traduce en respuesta de la Demanda (disminución 
de la cantidad demandada). Además, cuanto más elástica es la demanda (curva D'-D', mayor $\eta$ ), menores serán los incrementos de precio y mayores las reducciones de la cantidad demandada.

Ahora estamos en capacidad de enfatizar dos hechos interesantes.

o Si en un mercado, donde los productores del bien tienen la capacidad de prever y reaccionar ante cambios en la demanda, se produce una reducción de esta, es probable que la oferta se contraiga para compensar la reducción de precio. Más adelante se demostrará que los productores de energía en El Salvador, por la estructura del mercado eléctrico, tienen una gran capacidad de respuesta ante variaciones de la demanda.

o Si en un mercado, donde la demanda es muy elástica, se produce una contracción de la oferta, se producirá un alza de precio menor que la hubiese ocurrido si la demanda fuese muy inelástica. En el anexo 1 se demuestra mediante un modelo econométrico que la demanda de energía eléctrica en El Salvador es sumamente inelástica respecto del precio.

\section{La demanda de energía eléctrica en El Salvador}

Existe bastante evidencia empírica ${ }^{7}$ internacional que respalda el hecho de que la demanda de energía eléctrica, por su naturaleza, es muy poco sensible a las variaciones de precio; o que es muy "inelástica". Esto es así debido a que, si bien es cierto que los demandantes de energía estarían dispuestos a reducir su consumo al aumentar el precio, existen importantes costos de ajuste y efectos de saturación que evitan que la demanda de energía reaccione en el corto plazo. Esta propiedad, sin embargo, es correcta en un horizonte de tiempo muy corto; sobre plazos mayores es probable que los consumidores de energía reemplacen sus equipos por otros más eficientes o que cambien sus patrones de consumo.

En el caso de El Salvador no hay razones para pensar que la situación sería diferente.

Un modelo de demanda de energía eléctrica

La demanda global de energía eléctrica, para El Salvador, está compuesta por los consumos en cuatro rubros principales: Residencial, industrial, comercial y gobierno. 
El patrón de consumo residencial de energía eléctrica está influenciado principalmente por el modus vivendi de la población y tiene que ver tanto con los horarios laborales como con patrones culturales; sin embargo la cantidad de energía consumida en un hogar está más relacionada con el nivel de ingresos del hogar, del tamaño del mismo, con la temperatura ambiente ${ }^{8}$ y con cierto comportamiento estacional. Ahora bien estamos interesados en caracterizar el consumo de energía de todo el parque residencial de modo que habrá que hacer algunas consideraciones especiales. El nivel de ingresos tiende a explicar el consumo de energía (de cualquier tipo) ${ }^{9}$ en un análisis entre categorías, pero, a priori, no hay razón para esperar que el ingreso agregado explique el consumo energético a nivel residencial, al menos no en el corto plazo. Más probable es que el incremento en consumo de energía se deba a incrementos en la temperatura ambiente puesto que los equipos de refrigeración consumen más energía cuanto más grande es la diferencia de temperatura con el medio ambiente. Otro factor importante es la población, pero por ser el nuestro un análisis de corto plazo no consideraremos esa variable en nuestro modelo. Un último factor que pudiese afectar el consumo de electricidad para uso residencial es el que ocurran fechas o eventos especiales que afecten el tiempo de permanencia en el hogar y el aumento en el uso de energía. El precio relativo de la energía, tal como lo perciben las familias pudiese ser relevante según la teoría económica; sin embargo debido a que el consumo eléctrico está caracterizado por costumbres arraigadas y por altos costos de ajuste es probable que su importancia sea menor.

La demanda de energía para uso industrial está afectada principalmente por el nivel de actividad económica ${ }^{10}$, de modo que un buen indicador debiese ser el índice de variación de la actividad económica (IVAE) o el índice de Volumen de la Producción Industrial (IVOPI), publicados por el Banco Central de Reserva de El Salvador. Dado que el IVAE también recoge información de actividades poco intensivas en el uso de energía eléctrica se privilegiará el IVOPI como indicador de la actividad en este sector.

La demanda de energía para comercios está predominantemente influenciada ${ }^{11}$ por el nivel de actividad económica ya que eso afecta el número de horas laborales por día en ese sector y con ello su consumo de energía. Un índice apropiado sería el Índice de Volumen de la Actividad Comercial (IVAC). También está influenciada por la tem- 
peratura ambiente debido al aumento en el consumo energético de los equipos de acondicionamiento de ambientes.

Finalmente, la demanda energética del gobierno está compuesta principalmente por el consumo en todas las instalaciones y/o oficinas del Estado ${ }^{12}$. Las actividades del Estado se realizan de manera muy uniforme a lo largo de todo el año. Esto lleva a pensar que su consumo estaría muy poco influido por variables de entorno excepto la temperatura ambiente que afectaría los equipos de acondicionamiento de ambientes.

Entonces una función estimable de demanda de energía eléctrica sería:

$$
E_{\text {dem }, t}^{\circ}=f\left(\begin{array}{c}
P_{t}^{E} \\
I P C_{t}
\end{array}, I V O P I_{t}, I V A C_{t}, T_{t}^{\circ}\right)
$$

donde $P_{t}^{E}$ es el precio relativo de la energía eléctrica medid como el cociente del precio promedio de las empresas distribuidoras (tarifa eléctrica) y el índice de precios al consumidor del período en estudio. IVOPI es el índice de volumen de la producción industrial, IVAC es el índice e variación de la actividad Comercial, $T^{o}$ es la temperatura ambiente promedio del mes en los centros de carga.

Los datos disponibles imponen ciertas limitantes. Los datos del IVOPI y el IVAC se publican mensualmente y con un rezago de aproximadamente un mes.

La temperatura ambiente es diferente en cada centro de carga, de modo que se utilizará un "proxi", que es la temperatura promedio mensual del área metropolitana de San Salvador.

Los pliegos tarifarios de las distribuidoras han cambiado en períodos irregulares y además son distintos para cada una, lo que obliga a construir un precio promedio para el consolidado.

El resultado final es una muestra de 56 datos, de enero- 98 hasta Agosto-2002, para las cuatro variables en cuestión.

Como lo que necesitamos es establecer el valor de la elasticidad precio de la demanda, entonces la ecuación econométrica sería:

$$
\operatorname{Ln}\left(E_{d e m, t}^{\circ}\right)=C+\beta_{1} \operatorname{Ln}\left(\begin{array}{c}
P_{t}^{E} \\
I P C_{t}
\end{array}\right)+\beta_{2} \operatorname{Ln}\left(I V O P I_{t}\right)+\beta_{3} \operatorname{Ln}\left(I V A C_{t}\right)+\beta_{4} \operatorname{Ln}\left(T_{t}^{\circ}\right)
$$


Donde el valor de $\beta_{1}$ será igual al valor estimado de la elasticidad precio de la demanda de energía. Los resultados de la estimación son los que aparecen en la tabla 1. Esta ecuación presenta algunos problemas prácticos dado que algunas de las variables presentan tendencia y otras tienen comportamientos alrededor de una media fija. Además la variable IVAC y la variable IVOPI tienen una correlación muy cercana y aparece un problema a la hora de la estimación del modelo. Para eso se optó por el método de estimación en primeras diferencias que consiste en estimar una ecuación como la siguiente:

$$
\begin{aligned}
& \operatorname{Ln}\left(E_{\text {dem, }, t}^{\circ}\right)-\operatorname{Ln}\left(E_{d e m, t-1}^{\circ}\right)=\alpha+\gamma_{1}\left(\operatorname{Ln}\left(\begin{array}{c}
P_{t}^{E} \\
I P C_{t}
\end{array}\right)-\operatorname{Ln}\left(\begin{array}{c}
P_{t-1}^{E} \\
I P C_{t-1}
\end{array}\right)\right)+\gamma_{2}\left(\operatorname{Ln}\left(I V O P I_{t}\right)-\operatorname{Ln}\left(I V O P I_{t-1}\right)\right) \\
& +\lambda 3\left(\operatorname{Ln}\left(T_{t}^{\circ}\right)-\operatorname{Ln}\left(T_{t-1}^{\circ}\right)\right)
\end{aligned}
$$

La interpretación de los coeficientes es la misma que la de los coeficientes de la ecuación anterior. Los resultados de la estimación son los que aparecen en la tabla 2.

\begin{tabular}{|c|c|c|c|c|}
\hline $\begin{array}{l}\text { Dependent Variable } \\
\text { Method: Least Squa } \\
\text { Sample: 1998:01 } 20 \\
\text { Included observatio }\end{array}$ & EMANDA & & & \\
\hline Variable & Coefficient & Std. Error & t-Statistic & Prob. \\
\hline $\mathrm{C}$ & 0.414764 & 0.521195 & 0.795795 & 0.4298 \\
\hline IVOPI & 0.160530 & 0.048292 & 3.324189 & 0.0016 \\
\hline PRECIO & -0.050610 & 0.038628 & -1.310174 & 0.1959 \\
\hline TEMPER & 0.409502 & 0.112734 & 3.632451 & 0.0006 \\
\hline R-squared & 0.369896 & Mean de & ndent var & 2.356018 \\
\hline Adjusted R-squared & 0.333544 & S.D. dep & dent var & 0.043217 \\
\hline S.E. of regression & 0.035281 & Akaike & criterion & -3.782193 \\
\hline Sum squared resid & 0.064727 & Schwar: & iterion & -3.637525 \\
\hline Log likelihood & 109.9014 & F-statist & & 10.17535 \\
\hline Durbin-Watson stat & 0.563734 & Prob(F- & istic) & 0.000022 \\
\hline
\end{tabular}

Tabla 1 


\section{Tabla 2}

\begin{tabular}{|c|c|c|c|c|}
\hline \multicolumn{5}{|c|}{$\begin{array}{l}\text { Dependent Variable: D(DEMANDA) } \\
\text { Method: Least Squares } \\
\text { Sample(adjusted): 1998:02 2002:08 } \\
\text { Included observations: } 55 \text { after adjusting endpoints } \\
\end{array}$} \\
\hline Variable & Coefficient & Std. Error & $\mathrm{t}$-Statistic & Prob. \\
\hline $\begin{array}{c}\text { C } \\
\text { D(IVOPI) } \\
\text { D(PRECIO) } \\
\text { D(TEMPER) }\end{array}$ & $\begin{array}{l}0.001910 \\
0.108086 \\
-0.063490 \\
0.308430\end{array}$ & $\begin{array}{l}0.003528 \\
0.040973 \\
0.029060 \\
0.102389\end{array}$ & $\begin{array}{l}0.541445 \\
2.637965 \\
-2.184760 \\
3.012340\end{array}$ & $\begin{array}{l}0.5906 \\
0.0110 \\
0.0335 \\
0.0040\end{array}$ \\
\hline $\begin{array}{l}\text { R-squared } \\
\text { Adjusted R-squared } \\
\text { S.E. of regression } \\
\text { Sum squared resid } \\
\text { Log likelihood } \\
\text { Durbin-Watson stat }\end{array}$ & $\begin{array}{l}0.309397 \\
0.268773 \\
0.026148 \\
0.034870 \\
124.4533 \\
2.526697 \\
\end{array}$ & \multicolumn{2}{|c|}{$\begin{array}{l}\text { Mean dependent var } \\
\text { S.D. dependent var } \\
\text { Akaike info criterion } \\
\text { Schwarz criterion } \\
\text { F-statistic } \\
\text { Prob(F-statistic) }\end{array}$} & $\begin{array}{l}0.002433 \\
0.030579 \\
-4.380119 \\
-4.234132 \\
7.616164 \\
0.000266 \\
\end{array}$ \\
\hline
\end{tabular}

Luego, el efecto de los cambios de precio en la demanda de energía se estiman como el efecto del coeficiente, en la regresión en primeras diferencias, del precio de la energía.

El valor que se obtuvo fue de $-0.06349^{13}$. Este valor indica que por cada $1 \%$ de aumento del precio, la demanda se reduce un $0.06349 \%$. Esto indica que la demanda es muy poco sensible a las variaciones de precio y que, en cambio, es más sensible ante sus otros determinantes tal como lo muestran los coeficientes restantes (ver Anexo 1).

\section{La oferta de energía}

En la sección anterior se demostró que la demanda de energía es insensible a los cambios de precios en el corto plazo. Con eso se cumple una de las condiciones para que la iniciativa del "poder de la demanda" sea inefectiva. Sin embargo, todavía resta demostrar que se cumple la otra condición: que la oferta tenga la capacidad de reaccionar en el corto plazo ante variaciones en el entorno.

En la figura 4 se muestra la oferta y la demanda de un bien cualquiera. Puede verse que cuando se mueve la curva de oferta (de S-S a S'-S'), el punto de equilibrio del mercado se desplaza a lo largo de la curva de demanda que tiene pendiente negativa, por lo que el precio (P) y la cantidad transada (Q) se mueven en direcciones opuestas. Por 
tanto podemos afirmar que cuando el precio y la cantidad tranzada de un bien se mueven en sentidos opuestos se puede sospechar que ha ocurrido un desplazamiento de la oferta ${ }^{14}$.

\section{Figura 4}

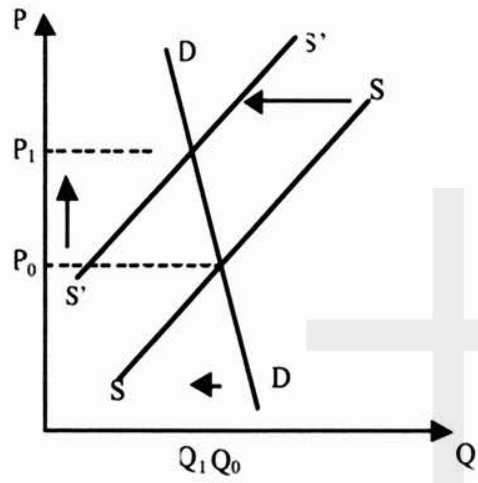

En la figura 5 se muestra la evolución de las tasas de cambio diarias, en el año 2003, de la Demanda de Energía Eléctrica y el precio Promedio de esta en el mercado Mayorista de Electricidad. Puede apreciarse a simple vista la mayor volatilidad de la tasa de cambio del precio, con variaciones que oscilan entre $164 \%$ y $-51 \%$, en tanto que la demanda experimenta variaciones de entre $43 \%$ y $-19 \%$. Pero más interesante es el hecho de que hay períodos en que el precio sube

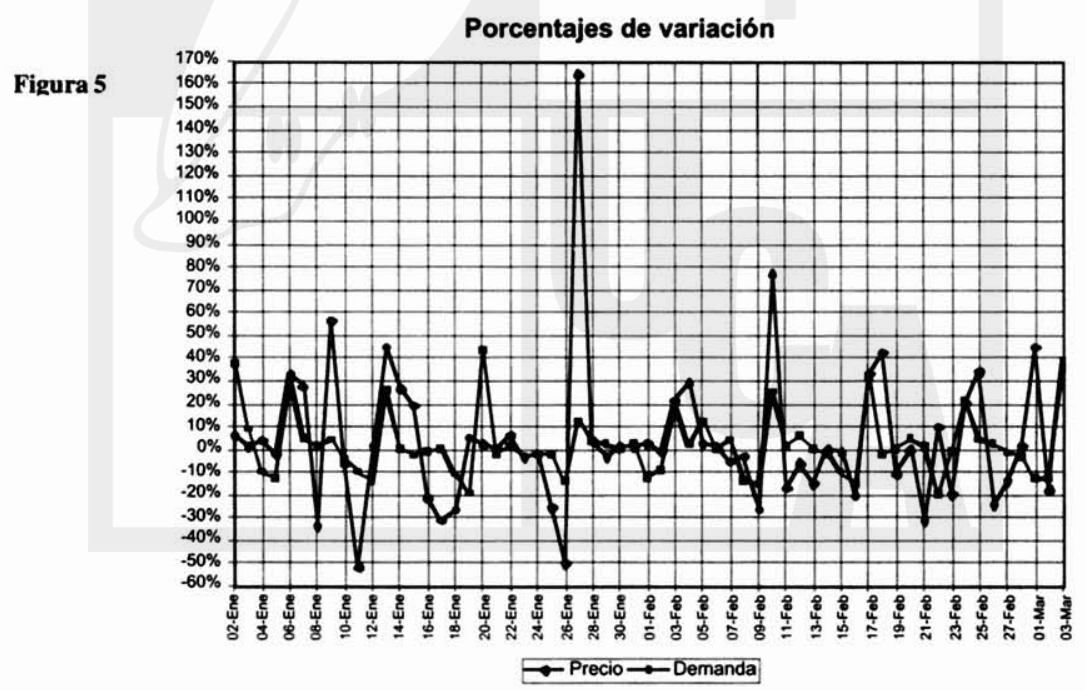

La falacia del poder de la demanda 
mientras la demanda cae y viceversa (valores de las variaciones con signos opuestos). De hecho eso sucedió en 21 de los 61 días mostrados del año 2003. Entonces, dada la periodicidad de la información y la frecuencia con que ocurren los eventos, puede verse que la reacción de la oferta es bastante rápida.

Resulta un poco más claro observar los gráficos construidos a partir de las declaraciones de las ofertas de oportunidad en el mercado Eléctrico mayorista de El Salvador. En las figuras de la 6 a la 8 podemos ver las ofertas de oportunidad del día 23 de Enero de 2002, que definen 24 curvas de oferta en un día ( 1 curva por hora). El color de las curvas determina la hora del día a la que pertenecen.

Figura 6

\section{Horas Valle}

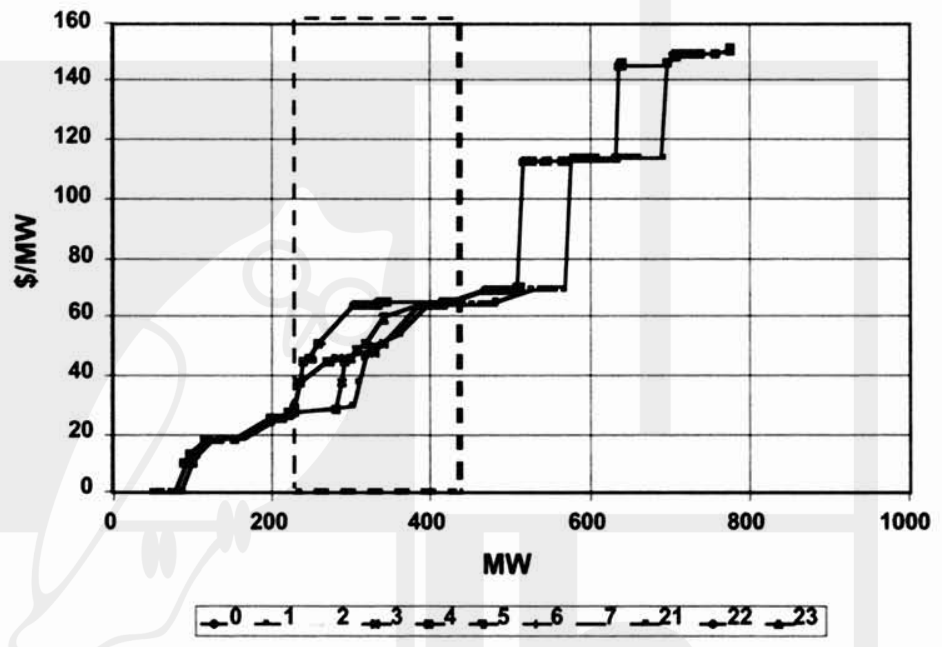

Las zonas sombreadas de los gráficos muestra el rango en que osciló la potencia transada en el mercado regulador del sistema en las horas en consideración. Definidas las curvas de Oferta, la potencia instantánea determina el precio del MWh en el Mercado mayorista de Electricidad, por ejemplo para las horas punta el precio fue de \$112.5/ MWh debido a que la demanda prevaleciente a esas horas fue superior a los $450 \mathrm{MW}$. 


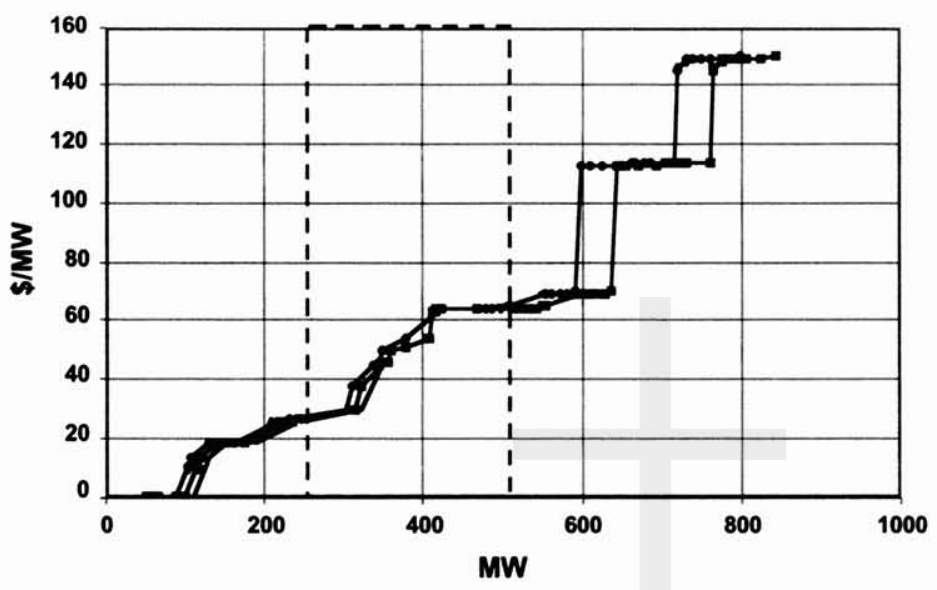

$\rightarrow 8-9+10 * 11-12 \rightarrow^{13} \rightarrow^{14}-15-16 \rightarrow 17$

Nótese que si este mismo nivel de demanda se hubiese registrado entre las 8:00 y las 17:59 el precio de la energía hubiese estado en un rango de entre $\$ 50 / \mathbf{M W h}$ y $\$ 70 / \mathbf{M W h}$. Si lo mismo se hubiese dado a las Horas del Valle, en cambo, el precio habría sido bien estable alrededor de \$115/MWh.

Figura 8

Horas Punta

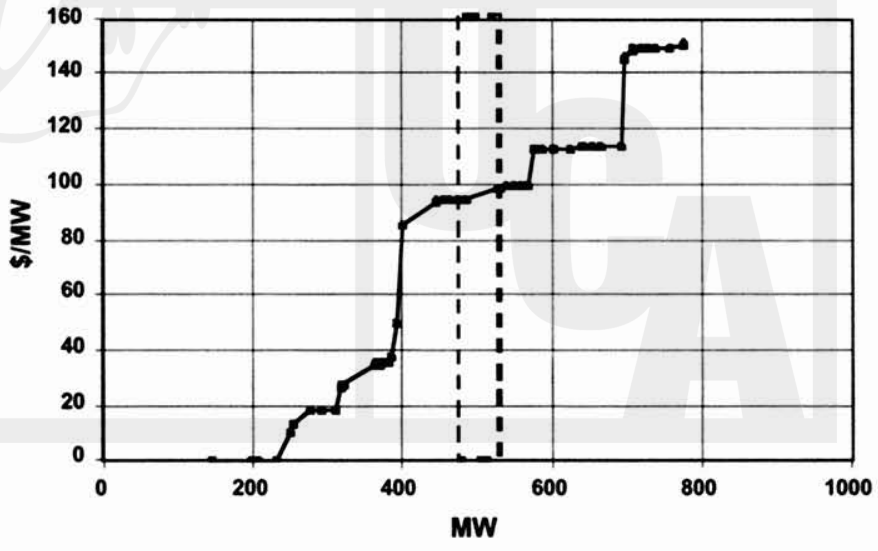

$\rightarrow-18+19+20$ 
En estos sencillos cuadros puede verse como la oferta reacciona incluso en períodos tan cortos como una hora.

Ahora cabe la pregunta sobre si ocurre lo mismo entre períodos más largos o solo es un fenómeno intradiario. Para responder esa cuestión debe plantearse un marco analítico inicial que nos permita contrastar nuestra hipótesis con los hechos.

En primer lugar, sabemos que la oferta puede variar debido principalmente a variaciones en los costos de producción; además, en el caso de los mercados con oferta muy concentrada (pocos oferentes en el Mercado) la oferta puede variar para cumplir con el objetivo de maximizar beneficios.

Los costos variables más importantes en la industria eléctrica son el costo del combustible y el costo de reposición del agua en los embalses de las presas hidroeléctricas; de ahí que puede esperarse que la oferta se contraiga en períodos en los que el precio del combustible sea elevado y/o cuando los embalses se encuentren en niveles bajos.

En la figura 9 se ve la evolución de la cota de los embalses de Cerrón Grande y Guija en los años 2001 y 2002. Se deduce entonces que los meses críticos son Mayo, Junio y Julio (principio de la época lluviosa); que son meses de bajo nivel de los embalses y en los que el costo de reposición del agua es mayor. En la figura 10 aparece la evolución del precio del petróleo West Texas Intermediate, tal como se cotiza en el New York Mercantile Exchange durante el año 2002.

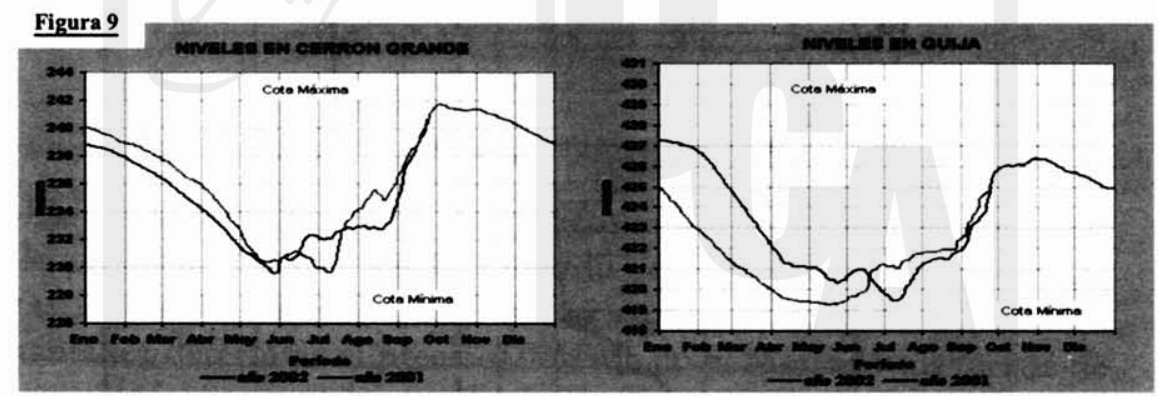




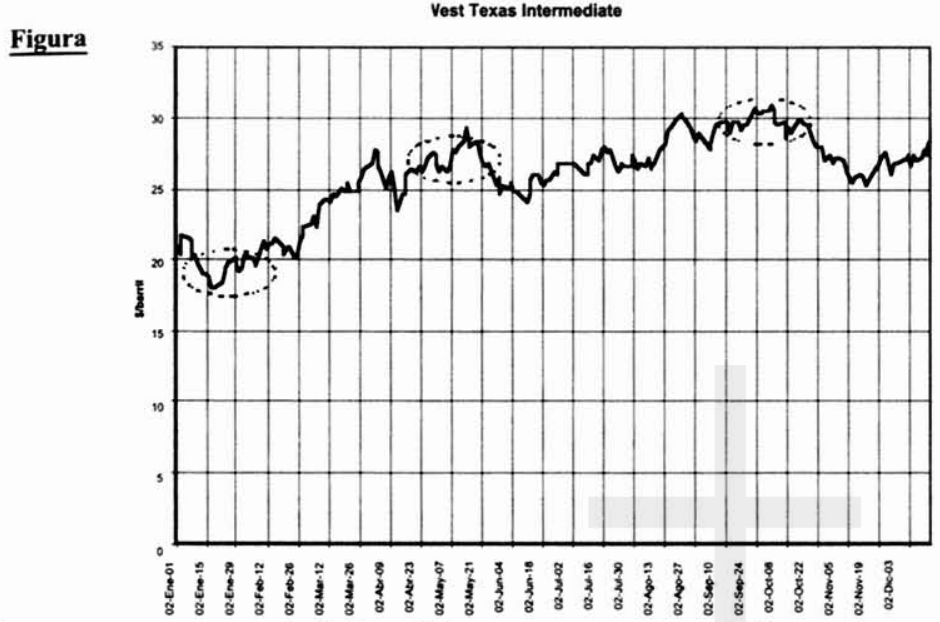

Observamos que a inicios del 2002 se registró el precio más bajo cerca de $\$ 18 /$ barril, pero se recupera rápidamente y alcanza un precio cercano a los $\$ 28 /$ barril a mediados de mayo. En los primeros días de Octubre el precio supera la barrera de los $\$ 30 /$ barril.

Con esto se tienen identificados tres posibles momentos de comparación de las ofertas del mercado regulador del sistema: El mes de enero con precio bajo del Petróleo y altos niveles de los embalses; El mes de mayo donde se registran precios altos del petróleo y las cotas más bajas de los embalses; finalmente, el período de mediados de septiembre a mediados de octubre donde el precio del petróleo es alto y los embalses están en sus niveles más altos.

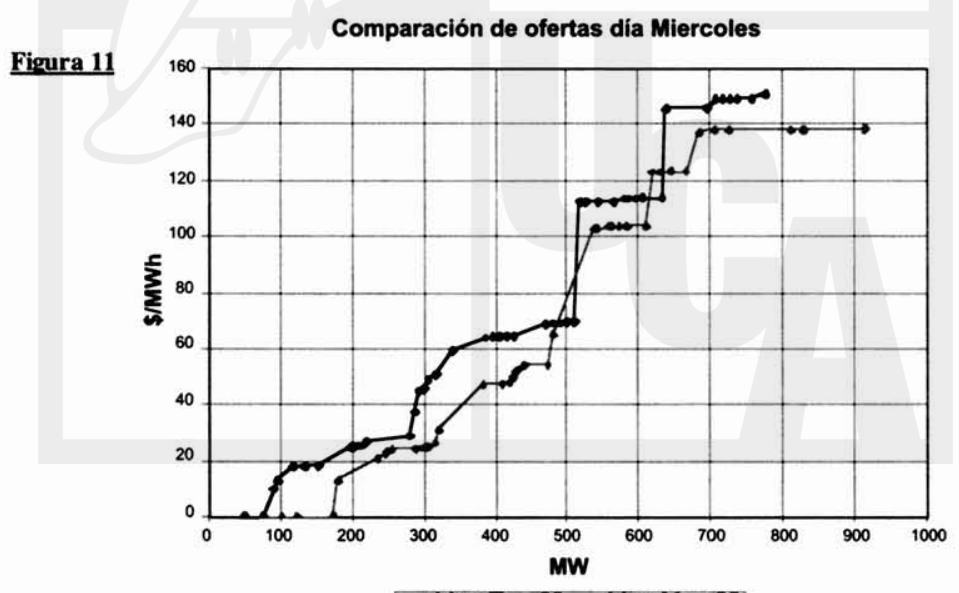

La falacia del poder de la demanda 
Figura 12

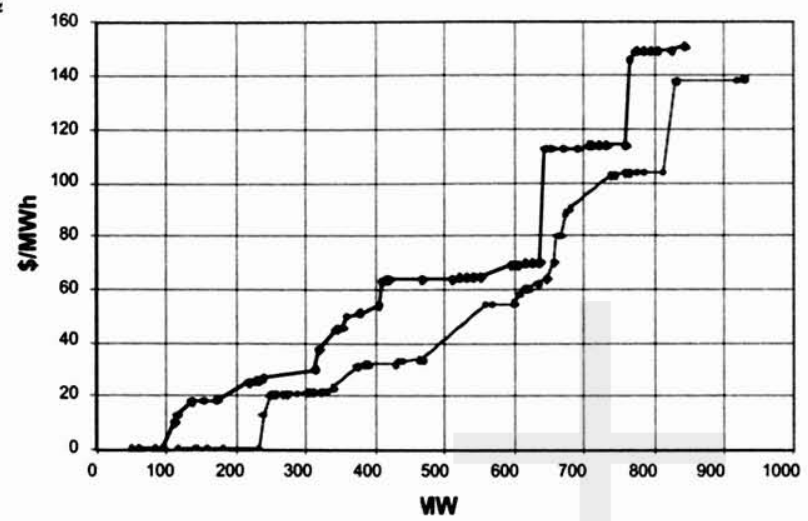

$\rightarrow$ hrs Ene-23 $\rightarrow$ hrs May-22

En enero, dadas las condiciones, se esperaría que la curva de oferta estuviese desplazada hacia adelante respecto de la curva de oferta de un mes como mayo en que las condiciones son más desfavorables. Sin embargo, en los gráficos 11 a 13 puede observarse que eso no sucede y que incluso las curvas de oferta del mes de enero están más contraídas que las de mayo; como si hubiesen bajado los costos de producción. Desde luego, esperar que la oferta se comporte acorde a los costos no es apropiado cuando se analiza un mercado que no tiene características competitivas ${ }^{15}$.

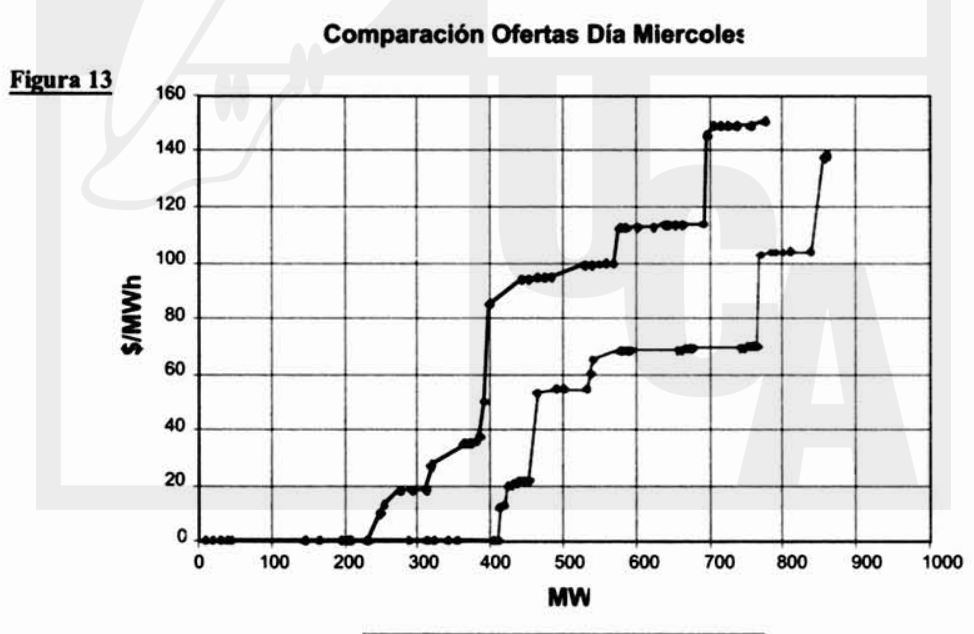

$\rightarrow 19$ hrs Ene-23 $\rightarrow 19$ Hrs May-22 
Los gráficos están construidos a partir de la curva de oferta ubicada más al centro del espectro de curvas en su rango horario particular, para un día laboral típico y con datos tomados de la UT.

Comparemos ahora las ofertas del 23 de enero con las del 02 de octubre (figuras de la 14 la 16). En cuestión de costos, la diferencia fundamental la hace el precio del petróleo, así pretendemos ver el impacto potencial del precio de los combustibles sobre las ofertas de energía.

\section{Figura 14 Comparación de Ofertas Día Miercoles}

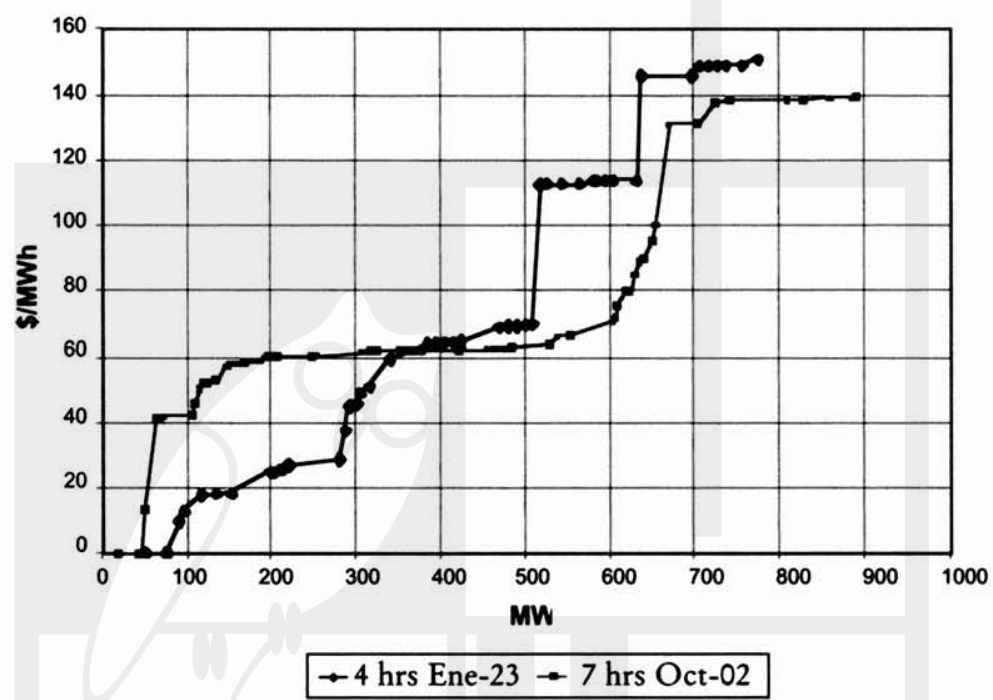

El movimiento de la curva de octubre respecto de la enero no es claro al analizar la curva completa pero se revela cuando consideramos los rangos de potencia que se tranzan típicamente a esas horas

Vemos que la curva correspondiente a las horas valle de octubre es la única que tiene un marcado recorte; sin embargo la curva de las horas resto no parece significativamente distinta para el rango de potencias en cuestión. Incluso la curva de la horas punta está por debajo de la curva de enero, contrario a lo esperado. 
Fiqure 15

Comparación de Ofertas Día Miercoles

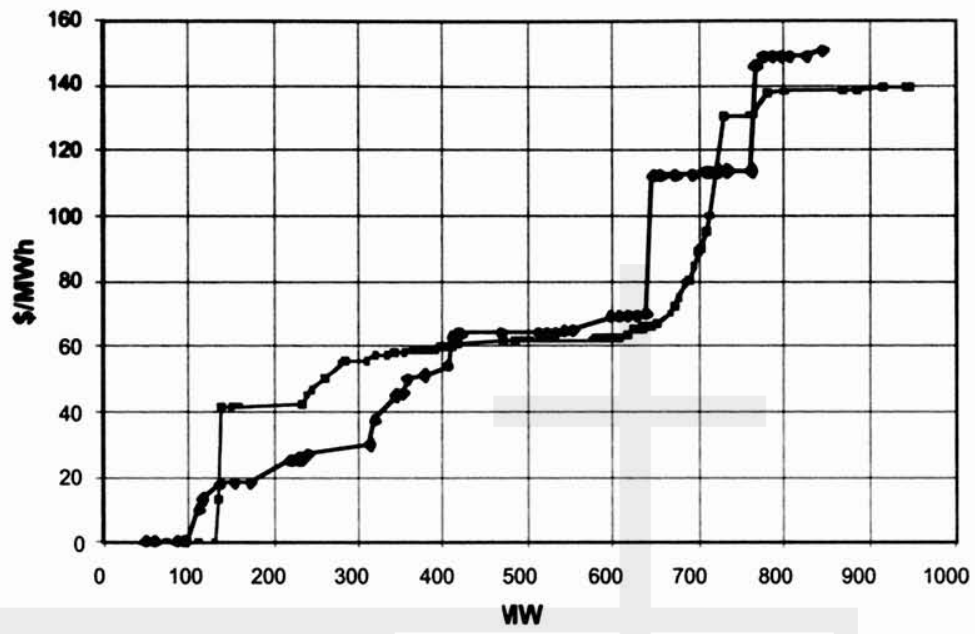

-9 hrs Ene-23 - 10 hrs Oct- 02

Comparación de Ofertas Día Miercoles

Figura 16

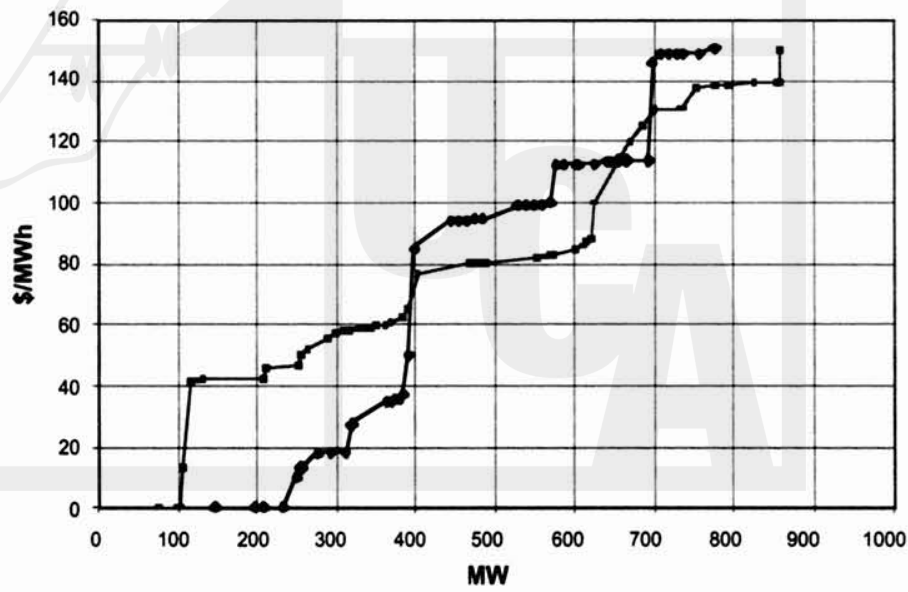

19 hrs Ene-23 -20 hrs Oct- 02 
Figura 17

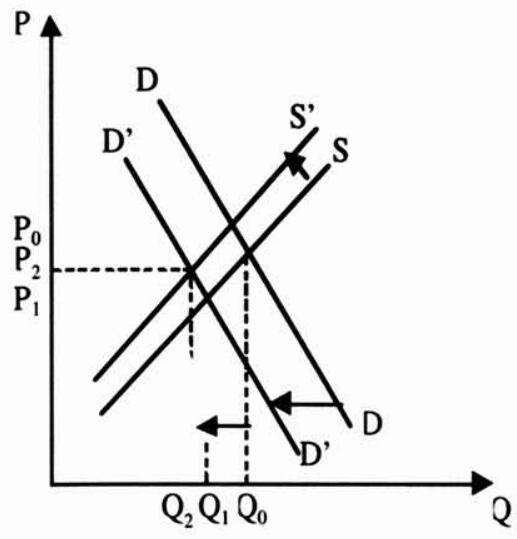

Aquí parece claro que la oferta eléctrica en el salvador, no solo no está directamente afectada por la estructura de costos de la industria, sino que tiene una gran capacidad de respuesta ante cambios en el entorno. Falta, ahora, analizar el comportamiento de esta ante reducciones en la demanda como las propuestas por la iniciativa del "poder de la demanda".

\section{Evaluación del Impacto de la Reducción de la Demanda}

La reducción de la demanda, como ya se ha visto, tendería a hacer bajar el precio del $\mathrm{MwH}$ y a reducir la cantidad tranzada de energía. Ahora se deben considerar dos posibilidades, que los oferentes sean sorprendidos por la reducción o que los oferentes la prevean y reaccionen para contrarrestarla. Como las declaraciones de las ofertas de oportunidad que determinan el precio del mercado regulador del sis-tema se hacen con un día de anticipación, es probable que el primer día de la reducción de demanda el precio de la energía se reduzca en alguna cantidad. Dicha reducción dependerá a su vez de lo significativa que sea la reducción en la demanda. Pero si ahora analizamos un horizonte de tiempo más largo veremos que la situación será muy diferente. Después del primer día de reducción, los agentes productores reaccionarán al descenso de la demanda de acuerdo con su expec-tativa sobre la duración de la disminución de la demanda. Si consideran que la reducción será transitoria y breve, probablemente decidan recortar la oferta ${ }^{16}$ en un monto tal que el precio baje menos de lo que bajo en las condiciones del primer día del recorte de demanda. (véase figura 17)

El punto $\left(Q_{0}, P_{0}\right)$ señala la cantidad y el precio de equilibrio en las condiciones iniciales. Cuando se reduce la demanda, el precio se reduce, hasta que el equilibrio de mercado ocurre en el punto $\left(\mathrm{Q}_{1}, \mathrm{P}_{1}\right)$. Recuérdese que este último punto sólo es posible el primer día de la reducción; al día siguiente, la oferta ha de reaccionar y se contraerá 
para hacer subir el precio hasta algún nivel $P_{2}$, por encima de $P_{1}$, que restablezca la condición de maximización de utilidades. Incluso es posible que al reducirse la demanda, el precio que se necesita para maximizar las utilidades de las empresas sea más alto que el precio inicial del mercado.

En conclusión, la reducción de la demanda tal como está descrita arriba no ayuda a bajar el precio de mercado dado que la oferta de energía reacciona con mucha prontitud a los cambios de la demanda.

Evaluación del impacto de la respuesta de la demanda ${ }^{17}$

Para evaluar el impacto potencial de la respuesta en demanda recuerde que sigue siendo válido que la oferta de energía puede reaccionar muy rápidamente. Es importante recordarlo ya que es posible que si la reacción de la oferta compensa las reducciones de la demanda, también lo haga con las respuestas de la demanda.

Es posible que los demandantes de energía realicen las inversiones necesarias para reaccionar a los cambios de precio en el mercado. Si esto ocurre y se sorprende a los productores de energía, es posible que el precio baje por un período corto de tiempo. Pero una vez que los productores reaccionen a la nueva situación de demanda ajustarán sus ofertas de modo que los precios no bajarán en la cuantía prevista inicialmente.

Pero aún si los productores no reaccionaran ante las respuestas de la demanda, no es claro que los beneficios de mejorar la respuesta en demanda sean grandes comparados con el costo de aumentar la respuesta de la demanda ${ }^{18}$. En la figura 18, podemos ver que durante un período de precio alto (debido a un recorte de la oferta desde $S_{0}-S_{0}$ hasta $S_{1}-S_{1}$ ) el incremento en precio fue de $P_{1}-P_{0}$ con la demanda inicial $\left(D_{0}-D_{0}\right)$; y de $P_{2}-P_{0}$ con una demanda más elástica $\left(D_{1}-D_{1}\right)$.

Bajo supuestos estándares, el beneficio neto del incremento de la respuesta en demanda es igual a la suma de las áreas $\mathrm{A}$ y $\mathrm{B}$ del gráfico 18. Esto es así, debido a que el costo de alcanzar el punto $\left(Q_{1}, P_{1}\right)$ de la situación inicial es $A+C$ (costo de generar $Q_{1}-Q_{2}$ ) más el $B+D$ (costo por dejar de consumir $Q_{0}-Q_{1}$ ), o sea $A+B+C+D$. En la situación donde mejoró la respuesta de la demanda, el costo de alcanzar el punto $\left(Q_{2}, P_{2}\right)$ es simplemente el costo por no consumir $Q_{0}-Q_{2}$, es decir $C+D$. Dado que queremos establecer el beneficio de

Realidad 92, 2003 
la situación final respecto de la inicial, calculamos la diferencia de costos como:

Beneficio $=$ Costo Inicial - Costo Final $=A+B$, que es el ahorro en costos debido a la mayor respuesta en demanda.

Figura 18

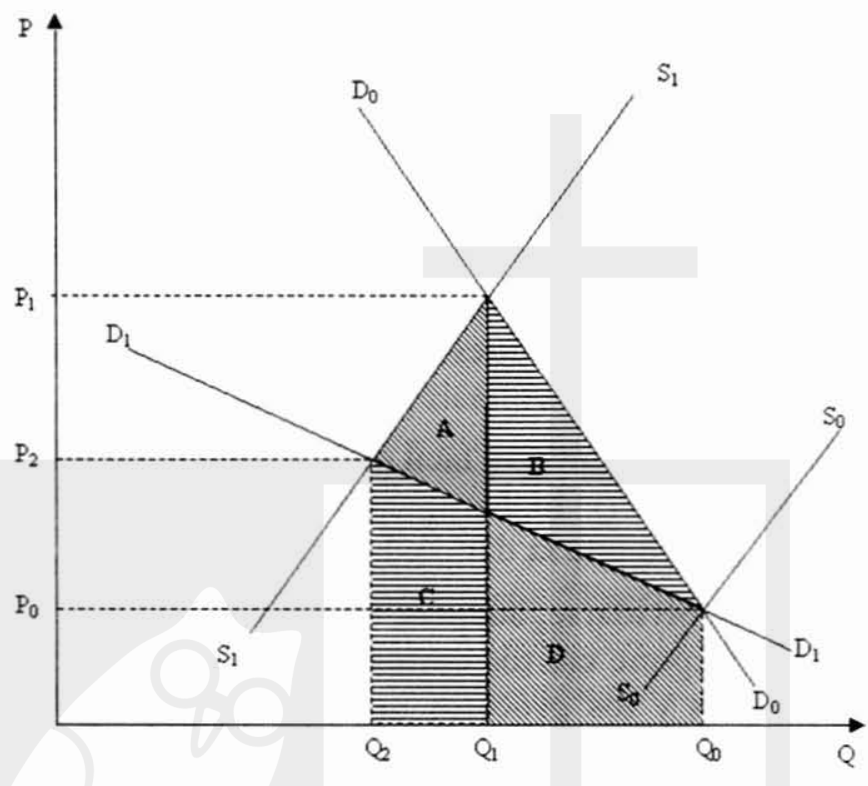

Pero, ¿que tan grande es este ahorro de costos? Si suponemos que la demanda inicial es completamente inelástica, el área del triangulo de ahorro de costos sería: $(1 / 2)\left(Q_{0}-Q_{2}\right)\left(P_{1}-P_{0}\right)$

Nótese que esta expresión dice que el beneficio de mejorar la respuesta en demanda depende del incremento de precios que hubiese ocurrido si no hubiese respondido la demanda, y de la reducción en la cantidad demandada de energía debido al aumento de precio; pero no de cuanto se reduzca el precio debido a la respuesta de la demanda.

El aumento en la factura total de energía debido al recorte de la oferta será de $\left(\mathrm{P}_{1}-\mathrm{P}_{0}\right) \mathrm{Q}_{0}$ (nótese que seguimos suponiendo que la demanda inicial es perfectamente inelástica). De modo que el ahorro en costos como fracción del aumento en la factura total es: $(1 / 2)\left(Q_{0}-\right.$ $\left.\mathrm{Q}_{2}\right) / \mathrm{Q}_{0}=0.5 \mathrm{X}$ donde $\mathrm{X}=\left(\mathrm{Q}_{0}-\mathrm{Q}_{2}\right) / \mathrm{Q}_{0}$ es igual al porcentaje de reducción de la demanda en respuesta al aumento de precio. Así una

La falacia del poder de la demanda 
reducción de la demanda del $5 \%$ traerá un ahorro en costos de tan solo el $2.5 \%$. Puede verse ahora que ante pequeños aumentos en la respuesta de la demanda, el ahorro de costos no será grande sino que será aún más pequeño en términos porcentuales.

\section{Conclusiones}

Hasta ahora ya se ha comprobado que existen las condiciones necesarias para que la iniciativa del "poder de la demanda" sea inefectiva, a saber:

- La demanda es casi completamente insensible a los cambios de precio en el corto plazo, $y$

- La oferta, en cambio, es extremadamente "ágil" y es capaz de responder a las variaciones del entorno en tiempos tan cortos como un día.

Con esto en mente, es fácil deducir que si la demanda se contrae, la oferta puede (y lo hará) contraerse también, compensando el efecto en precio que la demanda pretendía lograr.

No debe olvidarse, además, que la reducción y/o respuesta de la demanda impone ciertos costos, que derivan de los cambios en las instalaciones y los procesos necesarios para lograrla. Estos costos no son menores y probablemente la empresa que incurra en ellos no verá redituada su inversión en la cuantía inicialmente estimada ya que no habrá ningún efecto sobre el precio de la energía sino sólo sobre la cantidad de energía consumida.

Otro asunto importante es el hecho de que las reducciones de precios no son deseables per se, sino en la medida en que signifiquen ahorro de costos y no solo transferencias de rentas.

Una política encaminada a bajar los precios de la energía eléctrica debería, entonces, estar encaminada a eliminar las condiciones que limitan la competencia en la generación de energía o en su defecto simular el resultado competitivo mediante adecuada regulación. 
- Vivien Foster, Jean-Phillippe Tre \& Quentin Wodon. "Energy Consumtion and Income : an inverted- $U$ at the household level?”. World Bank. CTI Institute. Septiembre 2000. Hipervinculo "http://www.worldbank.org/wbi/B-SPAN/docs/ Gua_InvU2.pdf” www.worldbank.org/wbi/B-SPAN/docs/ Gua_InvU2.pdf

- Robert H. Patrick, Frank A. Wolak. "Estimating the CustomerLevel Demand for Electricity under Real-Time Markets Prices" NBER. Working paper 8213. Abril 2001. Hipervinculo "http:// www.nber.org/papers/w8213.pdf" www.nber.org/papers/ w8213.pdf

- Larry E. Ruff. Economic Principles of Demand Response in Electricity. Edison Institute. Septiembre 2002. Hipervínculo "http://www.stoft.com/e/lib/papers/Ruff-2002-demandresponse.pdf" http://www.stoft.com/e/lib/papers/Ruff-2002demand-response.pdf

- Hugh Rudnick, Juan-Pablo Montero. "Precios Eléctricos Flexibles" Cuadernos de Economía. Año 38. No.113 Abril 2001. Hipervinculo"http://www.ingenieriaindustrial.cl/spanish/ working_papers/draftce2.pdf" http://www.ingenieriaindustrial.cl/ spanish/working_papers/draftce $2 . p d f$

- Red Eléctrica de España. “Atlas de la Demanda Eléctrica Española". Proyecto INDEL. Hipervínculo "http://www.ree.es/ cap07/pdf/indel/Atlas_INDEL_REE.pdf" http://www.ree.es/ cap07/pdf/indel/Atlas_INDEL_REE.pdf

- William H. Green. Análisis Econométrico. Tercera edición. Prentice Hall Iberia. Madrid 1999. 
Las variables a incluir en el modelo, según se explicó son: IVOPI, IVAC, Precio de la Energía y Temperatura ambiente. Hay que hacer algunas aclaraciones respecto de los datos; el precio de la energía relevante para el modelo es el precio promedio de la energía a usuarios finales. Esto complica ligeramente el tratamiento ya que no existe un solo precio, de modo que se optó por construir un índice. Este índice se construyó como el promedio ponderado por participación en la demanda de los precios aplicables a las diferentes categorías tarifarias de las cuatro distribuidoras más importantes de El Salvador. Luego se procedió a deflactar este índice por la inflación de cada período para calcular el precio en términos reales.

La temperatura relevante para el modelo es la temperatura promedio en los centros de carga; esto, aunado al hecho de que los datos disponibles eran limitados, obligó a utilizar la temperatura promedio de la región metropolitana, que es el centro de carga más importante del país, calculada como la temperatura promedio de las estaciones meteorológicas cercanas a la zona.

Los datos se presentan en el siguiente anexo.

Así, el modelo de demanda se construyó según la siguiente ecuación:

$$
\operatorname{Ln}\left(E_{\text {dem, } t}^{\circ}\right)=C+\beta_{1} \operatorname{Ln}\left(\begin{array}{c}
P_{t}^{E} \\
I P C_{t}
\end{array}\right)+\beta_{2} \operatorname{Ln}\left(I V O P I_{t}\right)+\beta_{3} \operatorname{Ln}\left(I V A C_{t}\right)+\beta_{4} \operatorname{Ln}\left(T_{t}^{\circ}\right)
$$

de forma que los coeficientes se pueden interpretar como elasticidades de la demanda respecto a cada variable. En una primera estimación se obtuvo resultados poco satisfactorios debido a que las variables IVOPI e IVAC están muy correlacionadas, con lo que apareció el problema de la multicolinealidad. De ahí que se optara por estimar el modelo usando solamente el IVOPI que es la variable con mayor coeficiente de correlación respecto de la demanda de energía. 
Los resultados de la regresión fueron los siguientes:

\begin{tabular}{|c|c|c|c|c|}
\hline \multicolumn{5}{|c|}{$\begin{array}{l}\text { Dependent Variable: DEMANDA } \\
\text { Method: Least Squares } \\
\text { Sample: 1998:01 2002:08 } \\
\text { Included observations: } 56 \\
\end{array}$} \\
\hline Variable & Coefficient & Std. Error & t-Statistic & Prob. \\
\hline $\begin{array}{c}\text { C } \\
\text { IVOPI } \\
\text { PRECIO } \\
\text { TEMPER }\end{array}$ & $\begin{array}{l}0.414764 \\
0.160530 \\
-0.050610 \\
0.409502\end{array}$ & $\begin{array}{l}0.521195 \\
0.048292 \\
0.038628 \\
0.112734\end{array}$ & $\begin{array}{l}0.795795 \\
3.324189 \\
-1.310174 \\
3.632451\end{array}$ & $\begin{array}{l}0.4298 \\
0.0016 \\
0.1959 \\
0.0006\end{array}$ \\
\hline $\begin{array}{l}\text { R-squared } \\
\text { Adjusted R-squared } \\
\text { S.E. of regression } \\
\text { Sum squared resid } \\
\text { Log likelihood } \\
\text { Durbin-Watson stat }\end{array}$ & $\begin{array}{l}0.369896 \\
0.333544 \\
0.035281 \\
0.064727 \\
109.9014 \\
0.563734\end{array}$ & \multicolumn{2}{|c|}{$\begin{array}{l}\text { Mean dependent var } \\
\text { S.D. dependent var } \\
\text { Akaike info criterion } \\
\text { Schwarz criterion } \\
\text { F-statistic } \\
\text { Prob(F-statistic) }\end{array}$} & $\begin{array}{r}2.356018 \\
0.043217 \\
-3.782193 \\
-3.637525 \\
10.17535 \\
0.000022\end{array}$ \\
\hline
\end{tabular}

El bajo valor del estadístico Durbin-Watson indica correlación en los errores con lo que la inferencia quedaría invalidada. Un análisis del correlograma de los errores - Figura siguiente - demuestra que hay un problema en la estimación dado que los errores no son un ruido blanco:

Correlogram of Residuals

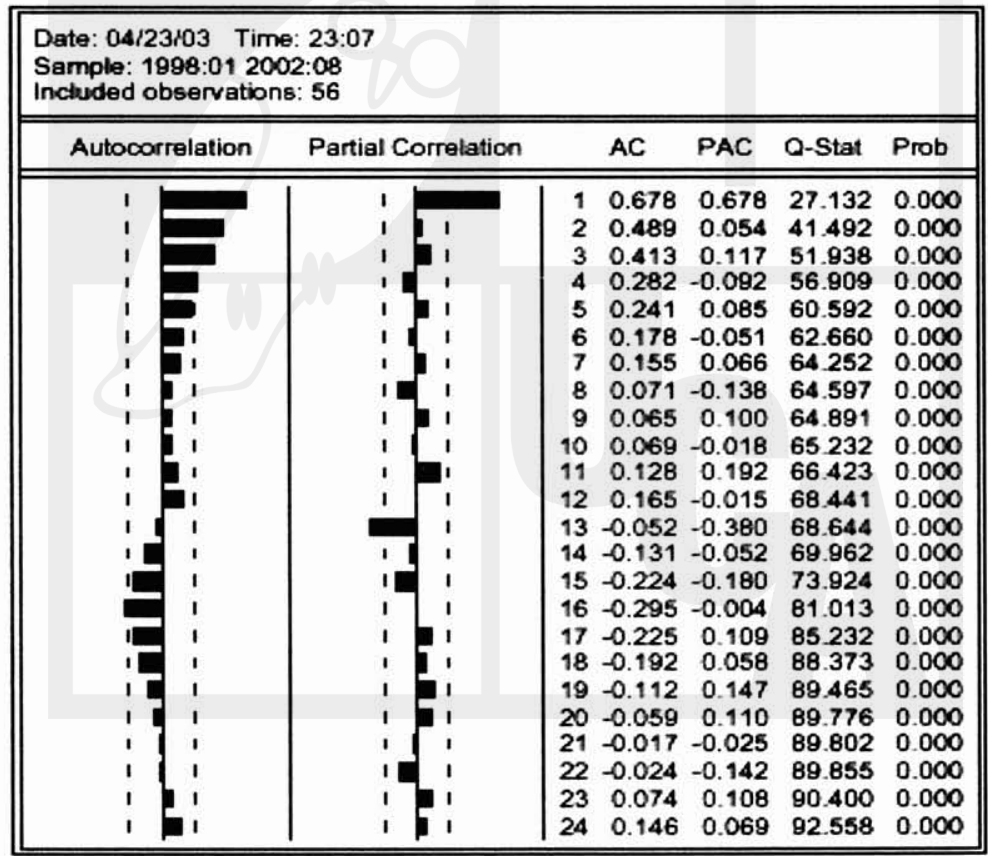


Dado lo anterior y pensando que probablemente el problema se deba a que algunas de las series no son estacionarias, se contrastó la existencia de raíces unitarias en las series:

\begin{tabular}{|c|c|c|c|}
\hline \multicolumn{4}{|l|}{ Demanda } \\
\hline ADF Test Statistic & 0.544291 & $1 \%$ Critical Value & -2.6048 \\
\hline & & $5 \%$ Critical Value & -1.9465 \\
\hline & & $10 \%$ Critical Value & -1.6189 \\
\hline \multicolumn{4}{|l|}{ IVOPI } \\
\hline \multirow[t]{3}{*}{ ADF Test Statistic } & -2.641853 & $1 \%$ Critical Value & -3.5547 \\
\hline & & $5 \%$ Critical Value & -2.9157 \\
\hline & & $10 \%$ Critical Value & -2.5953 \\
\hline \multicolumn{4}{|l|}{ Precio } \\
\hline \multirow[t]{3}{*}{ ADF Test Statistic } & -3.319026 & $1 \%$ Critical Value & -3.5547 \\
\hline & & $5 \%$ Critical Value & -2.9157 \\
\hline & & $10 \%$ Critical Value & -2.5953 \\
\hline \multicolumn{4}{|l|}{ Temperatura } \\
\hline \multirow[t]{3}{*}{ ADF Test Statistic } & -3.025092 & T\% Critical Value* & -3.5547 \\
\hline & & $5 \%$ Critical Value & -2.9157 \\
\hline & & $10 \%$ Critical Value & -2.5953 \\
\hline
\end{tabular}

Como vemos, solamente el precio y la temperatura resultaron ser estacionarias al $5 \%$ de significancia. Esto da lugar a que los errores no sean estacionarios o que no sean ruido blanco. Para intentar corregir este problema se realizó la misma regresión pero en primeras diferencias:

Dependent Variable: D(DDA)

Method: Least Squares

Sample(adjusted): 1998:02 2002:08

Included observations: 55 after adjusting endpoints

\begin{tabular}{lrlrr}
\hline \multicolumn{1}{c}{ Variable } & Coefficient & Std. Error & t-Statistic & Prob. \\
\hline C & 0.001910 & 0.003528 & 0.541445 & 0.5906 \\
D(IVOPI) & 0.108086 & 0.040973 & 2.637965 & 0.0110 \\
D(PRECIO) & -0.063490 & 0.029060 & -2.184760 & 0.0335 \\
D(TEMP) & 0.308430 & 0.102389 & 3.012340 & 0.0040 \\
\hline R-squared & 0.309397 & Mean dependent var & 0.002433 \\
Adjusted R-squared & 0.268773 & S.D. dependent var & 0.030579 \\
S.E. of regression & 0.026148 & Akaike info criterion & -4.380119 \\
Sum squared resid & 0.034870 & Schwarz criterion & -4.234132 \\
Log likelihood & 124.4533 & F-statistic & 7.616164 \\
Durbin-Watson stat & 2.526697 & Prob(F-statistic) & 0.000266 \\
\hline
\end{tabular}


En este caso el estadígrafo DF cae en la zona de indecisión, sin embargo una mirada al correlograma de los errores muestra que estos están mejor comportados:

Correlograma de los Residuos

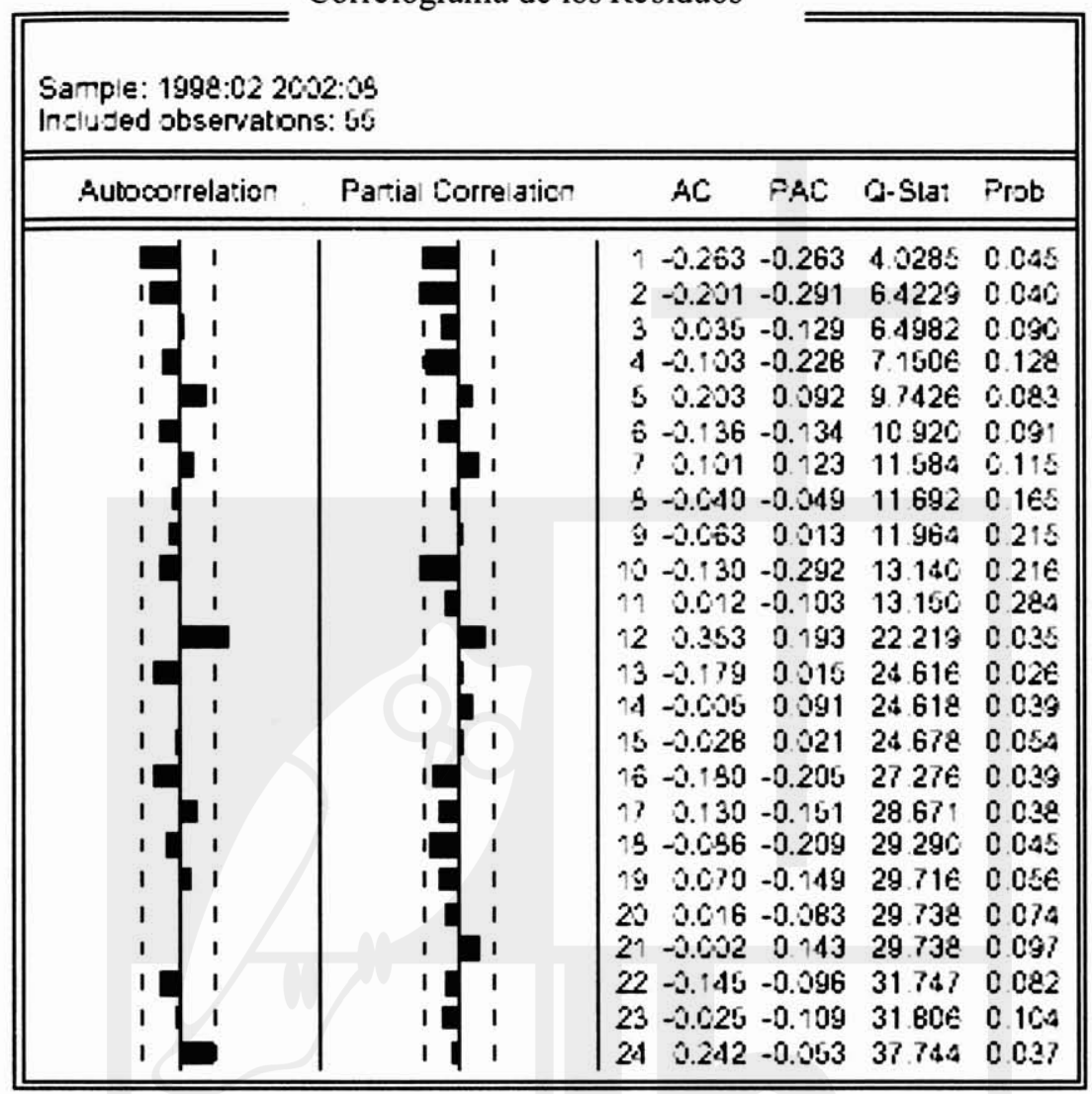

La falacia del poder de la demanda 
Finamente, vemos que los residuos soportan el contraste de normalidad de Jarque-Bera:

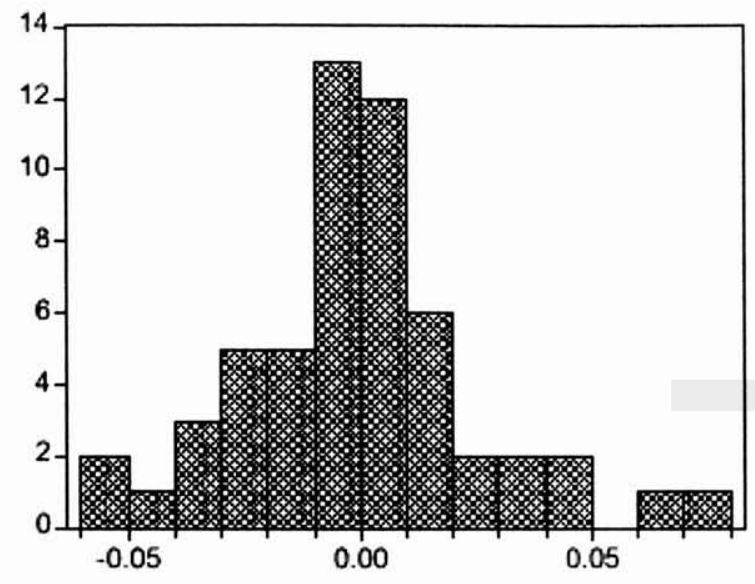

\begin{tabular}{|c|c|}
\hline \multicolumn{2}{|c|}{$\begin{array}{l}\text { Series: Residuals } \\
\text { Sample 1998:02 2002:08 } \\
\text { Observations } 55\end{array}$} \\
\hline \multicolumn{2}{|c|}{ Mean $-9.10 E-18$} \\
\hline $\begin{array}{l}\text { Median } \\
\text { Maximum }\end{array}$ & $\begin{array}{r}-0.000283 \\
0.072811\end{array}$ \\
\hline Minimum & -0.054307 \\
\hline $\begin{array}{l}\text { Std. Dev. } \\
\text { Skewness }\end{array}$ & \\
\hline Kurtosis & 3.928355 \\
\hline Jara & \\
\hline Probability & 0.161138 \\
\hline
\end{tabular}

Ahora ya podemos hacer alguna inferencia sobre los coeficientes de esta regresión y vemos que la demanda tiene una elasticidad precio de apenas -0.060349 , mientras que es más sensible a cambios en la temperatura y en la actividad económica. Vemos también que todos los coeficientes tienen el signo esperado y que las variables son significativas.

\section{NOTAS}

1. Horas de Mayor demanda en el sistema durante el primer semestre de 2002 según el informe semestral de la Unidad de Transacciones

2. La Ley general de Electricidad se publicó el 25 de octubre de 1996, en el diario oficial No. 201, tomo 333 y entró en vigencia 8 días después.

3. Se instaló Nejapa Power Plant propiedad, en ese tiempo, de Coastal Energy Group. 4. En épocas de bajo nivel de los embalses de las generadoras hidroeléctricas, es posible que estas también oferten precios altos.

5. En condiciones de congestión, la norma ha sido calcular un precio para cada zona en la que se separe el sistema. También puede ocurrir que en condiciones de falla exista más de un precio en una misma hora.

6. Cuando la cantidad demandada varía, no siempre es claro si es un desplazamiento de la curva de demanda o un movimiento a lo largo de la curva. La distinción es menos clara cuando se consideran distintos horizontes temporales. Por ejemplo, considérese un mercado de energía con precios "intradiarios" (Como el mercado eléctrico de El Salvador, donde el precio de la energía varía dentro de un mismo 


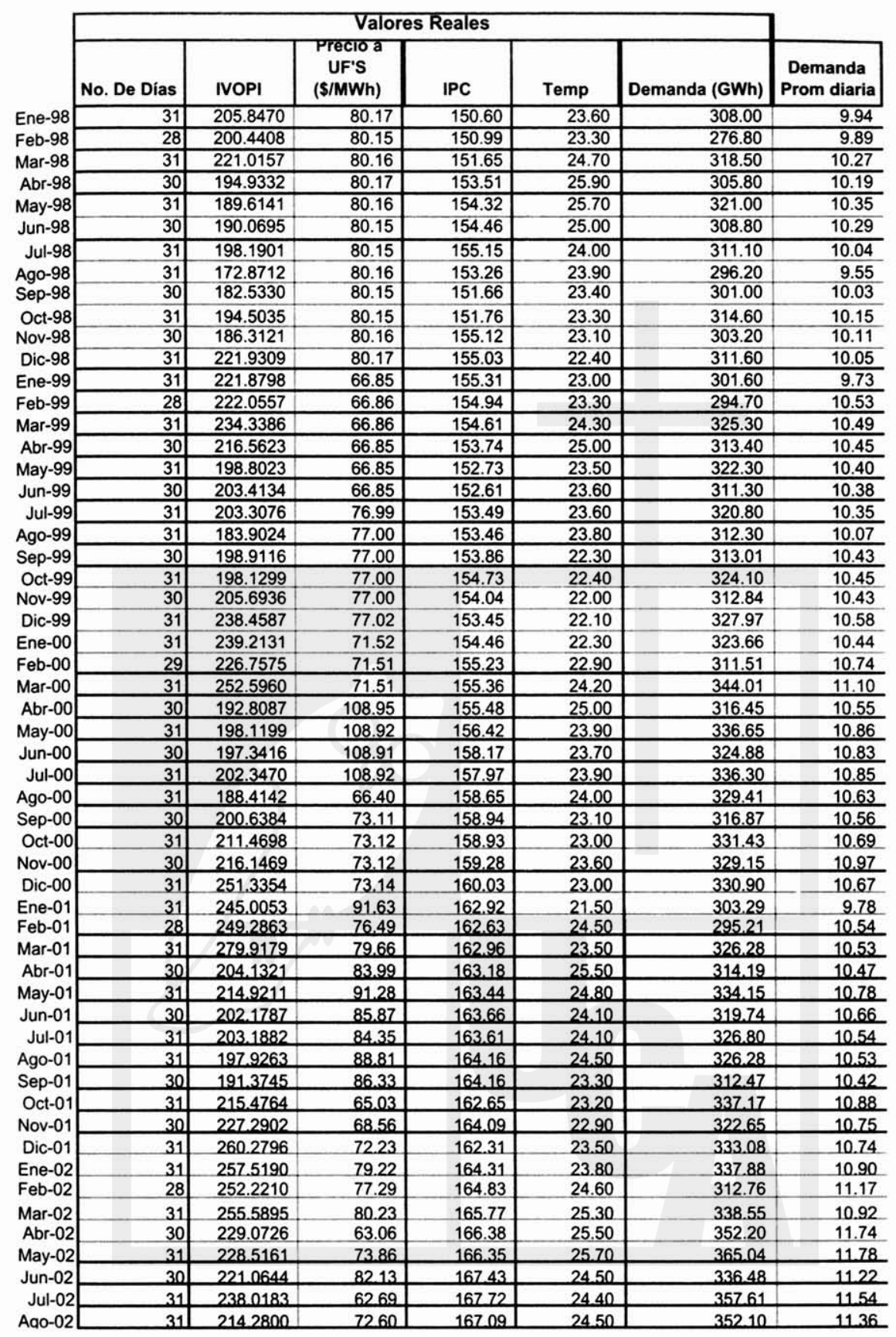


día); si en éste mercado se efectúan inversiones para reducir el consumo energético debido a que se prevé que el precio de la energía aumentará permanentemente, puede considerarse que ocurre un movimiento a lo largo de un curva de demanda de largo plazo. Pero si consideramos la misma reducción de demanda dentro del período de un día, diríamos que es un desplazamiento de una curva de demanda de corto plazo.

7. El autor no conoce de estudios serios para El Salvador en los que se caracterice la elasticidad de la demanda de energía eléctrica, sin embargo, puede hacerse referencia a estudios para otros países: Una caracterización de las elasticidades de demanda sectorial en Inglaterra se puede encontrar en Patrick \& Wolak (2001), También Rudnick \& Montero (2001) dan cuenta de diferentes valores de elasticidades de la demanda de energía en varios estudios, los valores cambian de acuerdo a la calidad de los datos, el número de observaciones y la técnica econométrica, pero, en general, los valores de elasticidad de corto plazo oscilan entre -0.11 y -1.01 .

8. En el estudio español de caracterización de la demanda de energía eléctrica residencial expuesto en el "Atlas de energía eléctrica" se establece que el consumo energético residencial está compuesto, en su utilización, principalmente por Calefacción, refrigeración, iluminación y otros electrodomésticos, en ese orden de importancia. Por obvias diferencias climáticas, en El Salvador no consideraremos el uso de electricidad para calefacción.

9. Foster, Tre \& Wodon (2000) explican, usando datos para Guatemala, la posible existencia de una " $U$ " invertida en el consumo de energía conforme aumenta el ingreso, debido a que al aumentar los ingresos de los individuos estos cambian de formas de energía ineficientes (leña, por ejemplo) a formas más eficientes (energía eléctrica), de forma que el consumo bruto de energía podría ser mayor en estratos inferiores de ingresos.

10. En realidad, para El Salvador, el autor no conoce ningún estudio al respecto, pero de todas formas lo que se intenta es determinar las principales determinantes de la demanda agregada de energía eléctrica y esta variable parece, a priori, relevante.

11. Véase nota 10

12. También podría considerarse la demanda de energía de ANDA pero para efectos de este estudio este consumo se considerará como exógeno.

13. Los detalles de las regresiones y los datos utilizados están en el Anexo 1.

14. El gráfico ilustra el caso más simple en el que solamente ha ocurrido un cambio paramétrico de la oferta

15. El índice Herfindal-Hirschman, que es una medida de la concentración de un mercado, ha oscilado entre 1988.06 y 2309.26 entre enero y diciembre de 2002 en el mercado de generación en El Salvador.

16. Las empresas hacen sus proyecciones de ingresos y costos suponiendo ciertas condiciones de demanda; cuando esas condiciones cambian, las ofertas se han de modificar para ajustar los ingresos a los nuevos costos de modo que las utilidades se maximicen nuevamente. Recuérdese que esto sólo es posible en mercados con características monopólicas u oligopólicas.

17. "The Economic Principles of Demand Response in Electricity", Larry E Ruff. Report to Edison Electric Institute. Septiembre 2002.

18. Se analiza el efecto del aumento de la respuesta en demanda desde la perspectiva del beneficio social debido a que las caídas de precio son en realidad transferencias netas de ingresos desde los productores hacia los consumidores y no puede argumentarse que son "deseables", ya que haría falta un argumento que indique que la situación después de la transferencia de renta es "mejor" que la situación inicial. 